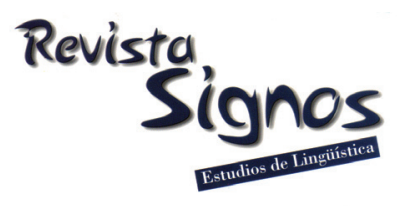

\title{
La Arquitectura de ELE-TUTOR: Un Sistema Tutorial Inteligente para el Español como Lengua Extranjera ${ }^{1}$
}

\author{
The Arquitecture of ELE-TUTOR: An Intelligent Tutorial \\ System for Spanish as a Foreign Language
}

\author{
Anita Ferreira \\ Universidad de Concepción \\ Chile \\ aferreir@udec.cl
}

\section{Gabriela Kotz}

Universidad de Concepción

Chile

gkotz@udec.cl

\author{
Pedro Salcedo \\ UNIVERSIDAD DE CONCEPCiÓN \\ Chile \\ psalcedo@udec.cl
}

\section{Fernanda Barrientos}

UnIVERSIDAD DE CONCEPCIÓN

Chile

fernandabarrientos@gmail.com

Recibido: 17-III-2011 / Aceptado: 20-X-2011

\section{Resumen}

Los Sistemas Tutoriales Inteligentes (STI) son aplicaciones computacionales utilizadas para la enseñanza de diversas disciplinas, entre ellas, la enseñanza de lenguas extranjeras. $\mathrm{Su}$ arquitectura se compone, principalmente de tres partes: un módulo del experto, un módulo del tutor y un módulo del estudiante, cada uno de estos componentes forman parte de un sistema mayor (STI) que, al articularse, permiten la adaptabilidad del sistema al conocimiento del estudiante. Para lograr una articulación completa del sistema, es necesario junto con diseñar el modelo de cada parte, también establecer los parámetros, variables e información que comparten cada uno ellos y la forma como llevan a cabo su tarea. En este trabajo se presenta la propuesta de un modelo de STI para la Enseñanza del Español como Lengua Extranjera, el diseño de cada módulo y los mecanismos como se articulan para conseguir el objetivo final del sistema, que es la adaptabilidad a las necesidades de cada estudiante en el contexto de la enseñanza del Español como Lengua Extranjera.

Palabras Clave: Español como Lengua Extranjera, Sistemas Tutoriales Inteligentes, parser, Procesamiento de Lenguaje Natural, Generación de Lenguaje Natural. 


\begin{abstract}
The Intelligent Tutorial Systems (ITS) are computer applications used for teaching diverse disciplines, among them for the area o foreign language teaching. The systems (ITS) consist of three main parts: an expert module, a tutor module, and a student module. Each of these components is part of a larger system (ITS) which allows the system to adapt to the learner's skill level. To achieve a complete articulation of the system it is necessary, besides designing the model for each component, to establish the parameters, the variables and the information shared by each of the components and the way they carry out the task. This paper presents the proposal of an ITS model for teaching Spanish as a foreign language, the design of each module, and the way the mechanism is articulated to achieve the final goal of the system, which is its adaptability as a tool for teaching Spanish as a foreign language, according to the students'needs.
\end{abstract}

Key Words: Spanish as a Foreign Language, Intelligent Tutorial Systems, parser, Natural Language Processing, Natural Language Generation.

\title{
INTRODUCCIÓN
}

Los Sistemas Tutoriales Inteligentes (STI) para lenguas extranjeras se basan en los desarrollos de la Inteligencia Artificial, principalmente, en lo que compete a la utilización de técnicas de Comprensión de Lenguaje Natural (del inglés, Natural Language Understanding) y de Generación de Lenguaje Natural (del inglés, Natural Language Generation). Un STI es un programa para la enseñanza-aprendizaje basado en el computador cuya finalidad última es la facilitación de los procesos de aprendizajes personalizados y autónomos. Estos sistemas son capaces de comportarse como un experto, tanto en el dominio de conocimiento que enseña como en el dominio metodológico, donde es capaz de diagnosticar la situación en la que se encuentra el estudiante $y$, de acuerdo con ello, ofrecer una acción o solución que le permita progresar en el aprendizaje (Ferreira, 2003).

A estos sistemas se les denomina 'inteligentes' atendiendo a la capacidad que ellos tienen para analizar gramaticalmente una entrada en lenguaje natural y luego producir un mensaje o enunciado en lenguaje natural correspondiente a una estrategia de feedback correctivo adecuado para el error focalizado del estudiante (Ferreira, Moore \& Mellish, 2007). Para ello, se debe implementar técnicas de Procesamiento de Lenguaje Natural (PLN) que se basan en teorías gramaticales para procesar la entrada del estudiante con el objeto de generar una estrategia de feedback. Las técnicas de PLN han permitido a los sistemas computacionales manejar algunas estrategias de feedback más sofisticadas como las claves metalingüísticas y los 'informes de errores’ basados en un análisis, identificación y jerarquización del error presentado en la respuesta del estudiante. 
Basados en investigaciones previas, proyectos Fondecyt 1040500 y 1080165 (Ferreira 2003, 2006, 2007; Ferreira, et al., 2007; Ferreira \& Kotz, 2010), se ha definido un modelo de tratamiento de los errores y un componente de estrategias de feedback. correctivo para un Sistema Tutorial Inteligente del Español como Lengua Extranjera, el cual toma en consideración el tipo de error gramatical que el estudiante comete y el nivel de aprendizaje (principiante, intermedio-avanzado). En el contexto de nuestra actual investigación, Proyecto Fondecyt 1110812, hemos continuado avanzando en la tématica de un tutor inteligente para el español como lengua extranjera, en lo que respecta a la configuración del diseño y desarrollo de los tres módulos de la arquitectura clásica de un STI, esto es, un tutor, un dominio y la definición del perfil del estudiante de Español como Lengua Extranjera (LE). De este modo, el propósito en este artículo es presentar la descripción de los módulos y sus componentes junto con la explicación de su articulación, todo ello con el objeto de sustentar la implementación de un STI que apoye el tratamiento de los errores gramaticales, adaptándose acorde con las características del modelo o perfil del estudiante de Español como Lengua Extranjera.

El artículo se organiza en las siguientes secciones: En la sección 1, nos referiremos a las principales investigaciones empíricas que se han llevado a cabo con respecto a los STI para lenguas extranjeras. En la sección 2, abordamos las técnicas de PLN en el contexto de analizadores y generadores automáticos de lenguas. En la sección 3, describimos la arquitectura del sistema tutorial inteligente ELE-TUTOR, especificando los componentes del modelo y la explicación de su funcionamiento. Por último, presentamos algunos comentarios finales y proyecciones sobre los avances y logros obtenidos en este estudio.

\section{Sistemas Tutoriales Inteligentes para la enseñanza de lenguas extranjeras}

El diseño de STI se basa en dos supuestos fundamentales sobre el aprendizaje. En primer lugar, la instrucción individualizada por un tutor competente es muy superior al estilo de aula tradicional, por cuanto tanto el contenido como el estilo de instrucción pueden ser continuamente adaptados para satisfacer de mejor forma las necesidades de la situación. En segundo lugar, los estudiantes aprenden mejor en contextos que se aproximen más estrechamente a aquellas situaciones en las cuales utilizarán sus conocimientos; es decir, aprenden haciendo, cometiendo errores, y construyendo el conocimiento de una manera más individualizada. Evidencia empírica ha demostrado que el método tutorial es superior a las experiencias de aprendizaje normales en ambientes de aulas tradicionales y se debe, principalmente, a los patrones de diálogo conversacionales (Graesser, Person \& Magliano, 1995) que facilitan el tratamiento de errores y corrección en el modo tutorial. Chi, Soler, Jeong, Yamauchi y Hausmann (2001) han sugerido que los estudiantes tienen mayores oportunidades de ser constructivos en interacciones con un sistema tutorial que en una sala de clases tradicional. 
El objetivo principal de un STI, es proporcionar ayuda en el proceso de la enseñanza y para que esto se pueda llevar a cabo de una manera efectiva y eficiente es necesario que este desempeñe algunas funciones importantes, como por ejemplo: interactuar con el estudiante por medio de un diálogo de iniciativa mixta, es decir, donde tanto el computador como el estudiante pueden iniciar preguntas y esperar respuestas razonables, indicar al estudiante las estrategias apropiadas para atacar un problema en particular y demostrarle las aplicaciones de estas estrategias en problemas concretos, responder al estudiante cualquier pregunta pertinente en términos comprensibles por este, decirle al estudiante no solo que el está errado e indicarle el método correcto de solución, sino también hacer hipótesis basadas en la historia de errores de este que es donde se encuentra la verdadera fuente de dificultades, ser capaz de resolver todos los problemas propuestos, si es posible de diferentes maneras, entender y criticar las soluciones encontradas por los estudiantes, crear un modelo del estudiante y modificarlo continuamente basándose en su desempeño y en reglas de enseñanza, dependiendo de lo que el estudiante demuestre que ha aprendido al resolver problemas que se le presentan, el sistema debe valorar lo hecho y tomar acciones que atiendan las deficiencias o los logros obtenidos, utilizar los errores de los estudiantes para diagnosticar sus fallas, ser capaz de combinar métodos algorítmicos y heurísticos.

La mayoría de las funciones antes mencionadas solo son posibles si estos sistemas generan y mantienen una imagen o modelo del estudiante, la cual se utiliza como base de todo su razonamiento y en consecuencia de su comportamiento. El modelo de estudiante es la imagen que el sistema tiene del estudiante, entendiendo el término imagen como posible interpretación de la realidad.

La Figura 1 muestra la estructura clásica de un STI genérico. Aparentemente, estos sistemas difieren poco de los sistemas CAI (Instrucción Asistida por Computador) que les preceden. En general, ambos se caracterizan por una filosofía común que incluye un gran control del tutor y un formato de tarea de respuesta corta. En ambos sistemas los estudiantes aprenden trabajando en series de cuestiones relativamente breves y en ambos casos el sistema juega exclusivamente el papel de experto en la tarea, controlando la selección de tareas o problemas, mientras que el estudiante es el responsable de resolverlos. El sistema también juega el papel de crítico, y en la mayoría de los STI es más el sistema que el estudiante quien decide cuándo debe proporcionarse una realimentación crítica. Las diferencias principales entre los STI y los primeros casi no reflejan diferencias en métodos de enseñanza ni filosofías de aprendizaje subyacentes sino logros de ingeniería y el procesamiento del lenguaje que permite al STI interactuar con el estudiante en una lengua determinada con el objeto de apoyar el proceso de enseñanza-aprendizaje. Además, al contrario que los primeros sistemas CAI, estos STI representan al menos parcialmente el conocimiento y razonamiento de un buen tutor humano individualizado y por tanto pueden apoyar de una forma más focalizada el aprendizaje individual de cada estudiante. 


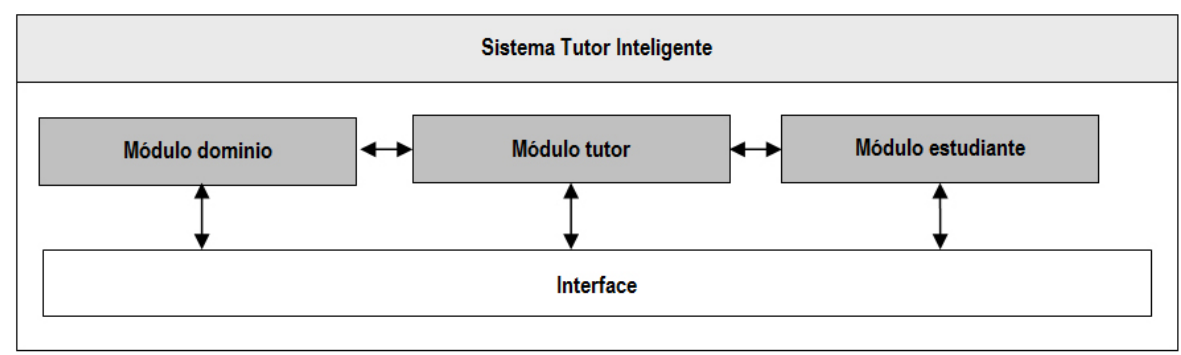

Figura 1. Arquitectura clásica de un STI propuesta por Carbonell (1970).

El núcleo de un STI es el módulo del tutor que incluye suficiente conocimiento sobre un área particular para proporcionar respuestas ideales a preguntas y corregir no solo un resultado final sino cada pequeña etapa de razonamiento intermedio. Esto permite mostrar y modelar una forma correcta de resolver un problema. Con frecuencia, como un tutor humano, puede generar muchos caminos de respuestas diferentes o 'estructuras objetivo'. Las mismas estructuras de datos detalladas que los sistemas expertos generan al modelar el razonamiento experto permiten explicar el razonamiento en niveles de detalle arbitrarios y por tanto monitorizar a un estudiante mientras resuelve un problema determinado, si cada etapa es correcta. Mientras que en los CAI la unidad atómica de discurso era la pregunta, aquí la unidad básica es la etapa de razonamiento individual. Para dar soporte a este entrenamiento paso a paso, con frecuencia crean y actualizan un modelo del estudiante, que refleja las reglas correctas que el STI cree que el estudiante conoce -algunas de las que se encuentran en el sistema experto o modelo de estudiante ideal. Cada vez que el estudiante comete un error, el STI diagnostica el problema - posiblemente actualizando el modelo del estudiante- y a continuación intenta remediarlo con un consejo muy detallado acerca de cómo el sistema experto habría operado en esta etapa. Este proceso se repite a cada paso en la evolución hacia la solución completa de un problema.

Un componente de la mayoría de los STI que recibe relativamente escasa mención es el módulo del dominio. Este contiene el conocimiento específico del área de enseñanza en cuestión, el que luego es contrastado con el del estudiante para poder estimar la diferencia y actualizar el modelo del alumno y sus creencias. Este modelo que requiere una buena representación formal de cada nodo de conocimiento y de sus enlaces, en diversas disciplinas y de una forma gráfica, es representado por mapas conceptuales o grafos. En los STI se ha preferido la representación de este conocimiento a través de redes semánticas complementadas con marcos, lo que permite un enlace de cada nodo y una descripción de cada uno de ellos. 


\section{Procesamiento de Lenguaje Natural (PNL): Análisis automático (parsing) y generación (generation) automática de lenguas en el contexto de STI}

Se han desarrollado pocos STI para la enseñanza de lenguas, ello originado, en parte, por las múltiples problemáticas relacionadas con la complejidad de los fenónemos en estudio (errores de lengua, estrategias de feedback, correctivo) y la falta de conocimiento acerca de las investigaciones en adquisición de segundas lenguas por parte de investigadores del área de los STI para LE (Ferreira, 2007; Ferreira et al., 2007).

Uno de los sistemas interesantes desarrollados en esta última década es el STI denominado Banzai para la enseñanza del japonés (Nagata, 2002). Este sistema emplea la tecnología de PLN con el objetivo de capacitar a los estudiantes para producir libremente frases en japonés y proporcionar un feedback detallado en lo que concierne a la naturaleza específica de los errores del estudiante. El tutor incorpora veinticuatro lecciones que cubren las construcciones gramaticales encontradas en un currículo de pregrado estándar. Dicho tutor acepta entradas en japonés, y presenta relevantes imágenes gráficas y fotográficas de Japón y de las situaciones cotidianas. Asimismo, Banzai permite al alumno producir cualquier frase ya que puede identificar las partes de la oración y los patrones sintácticos de las oraciones producidos por el alumno, sobre la base de los principios generales de la gramática japonesa. A la luz de estos principios gramaticales, Banzai determina si la frase es gramatical o agramatical y genera feedback inteligente dirigido a determinadas deficiencias en el rendimiento del estudiante.

Más recientemente, Heift (2003) y Heift y Schulze (2007) han desarrollado un STI para apoyar el proceso de aprendizaje del alemán como LE, el sistema E-TUTOR. Este sistema proporciona un feedback individualizado de errores específicos mediante la realización de un análisis lingüístico del imput del estudiante y un ajuste de los mensajes de retroalimentación adecuados a la habilidad de los alumnos. E-TUTOR también ofrece ayuda individualizada sensible al contexto, en función de los errores que los aprendices estén cometiendo. El sistema clasifica los errores del estudiante por medio del uso de una lista de prioridad flexible de error: las limitaciones de gramática pueden ser reordenadas a fin de reflejar el énfasis deseado de una actividad específica. La experiencia con E-TUTOR apoya la necesidad de un sistema tutorial que considere múltiples errores de acuerdo con la enseñanza de lenguas.

\subsection{Analizadores automáticos para la enseñanza de lenguas extranjeras}

El análisis automático (del término técnico en inglés parsing) es un proceso por medio del cual se convierte el texto de entrada en otras estructuras (comúnmente árboles sintácticos), que son más útiles para el posterior análisis y capturan la jerarquía 
implícita de la entrada. Durante el procesamiento se producen distintas estructuras intermedias o de trabajo, hasta producir un árbol de análisis estructural de la secuencia de entrada. Un árbol correcto es aquel que cubre todos y solo los elementos del enunciado y en cuyo tope tiene un símbolo S (del inglés sentence) u O (de 'oración') (Lavid, 2005; Jurafsky \& Martin, 2008).

Previo al procesamiento sintáctico de la oración, se deben cumplir varias etapas a fin de preparar el texto para el análisis. Las tareas básicas que se deben abordar en esta fase son: a) la segmentación del texto, b) el filtrado de información no relevante, y c) la localización de unidades tratables. A pesar de su aparente simplicidad, estas tareas pueden presentar algunas complicaciones en el caso que el texto no cuente con signos de puntuación, contenga errores o elementos que obstruyen el tratamiento del texto, como las marcas que definen las características de visualización de la página o los enlaces a otras páginas, en los casos de páginas de internet. Un problema adicional se presenta cuando hay distintos tipos de lenguas o grafías. En el caso de un analizador para la enseñanza/aprendizaje de lenguas extranjeras es que se manejan dos sistemas lingüísticos diferentes, una con el sistema de la lengua meta y otra con la 'interlengua' o las 'interlenguas' del alumno.

Se entiende por 'interlengua' a la competencia transicional que tiene el alumno en la lengua que está aprendiendo en un momento dado de su aprendizaje (Corder, 1981; Ellis, 1997; James, 1998) y cuyo conocimiento es fundamental para la detección automática de los errores por parte de un STI (Granger, 2003). A fin de obtener datos de la proficiencia del alumno en la LE, muchas veces previo a la interacción con el sistema se realiza un examen o evaluación en la LE.

Por lo general, un analizador automático no cubre la totalidad del sistema de la lengua, sino que se restringe al dominio de una 'sublengua' (Levin \& Evans, 1995) con las estructuras que se tratan en ese nivel de aprendizaje. En este contexto, muchos STI exitosos registran las acciones del analizador automático mediante la generación de reportes que permiten una actualización constante del módulo estudiante (Heift \& Schulze, 2007; Amaral \& Meurers, 2011).

En el ámbito de la interdisciplina lingüística Computer Assisted Language Learning (CALL), la importancia de los analizadores automáticos fue muy discutida en la última década por varios investigadores: Nagata, Matthews, Holland, Maisano, entre otros (en Heift \& Schulze, 2007; Schulze, 2008). Holland, Kaplan y Sams (1995) se refieren a las posibilidades y limitaciones de tutores de lengua basados en analizadores automáticos. Para ello, realizan una comparación entre sistemas CALL convencional y sistemas ICALL (Intelligent Computer Assisted Language Learning) basado en analizadores automáticos y concluyen que en sistemas ICALL el estudiante puede escribir una gran variedad de oraciones y desarrollar de una forma relativamente libre habilidades para el mejoramiento de la producción escrita. La eficacia de los analizadores automáticos en 
la enseñanza de lenguas fue analizada empíricamente por Jouzulynas (1994, en Heift \& Schulze, 2007) quien evaluó su utilidad en el diagnóstico de errores. Jouzulynas analizó errores en un corpus aproximado de 400 páginas de ensayos en alemán escritos por estudiantes norteamericanos en cursos de segundo año de lengua. Su estudio demuestra que el área de aprendizaje más problemático es la sintaxis, seguido por la morfología. Este estudio, que fue realizado con la ayuda de un analizador automático del alemán, indicó que la mayoría de los errores de los alumnos $(80 \%)$ no son de origen semántico y, por lo tanto, pueden ser reconocidos por un parser sintáctico. Jouzulynas adaptó la taxonomía de errores de Hendrickson (1979) que consiste en cuatro categorías: sintaxis, morfología, ortografía y léxico; clasificó los errores ortográficos en dos subcategorías: errores de escritura y de puntuación. Esta decisión está justificada en el contexto del sistema de parsing sintáctico. Los errores de puntuación contravienen reglas de sintaxis, los que consecuentemente podrían ser analizados por el analizador. Además, el analizador sintáctico se aplica normalmente en conjunto con un corrector ortográfico, por lo tanto, la mayoría de los errores ortográficos son subsanados antes de que el analizador automático entre en acción. En definitiva, ICALL basado en un analizador puede ser de gran utilidad para la detección de errores morfosintácticos que constituyen la mayoría de los errores que cometen los alumnos en la producción escrita libre.

Los analizadores diseñados para la enseñanza de lenguas, generalmente, contienen un componente que anticipa o detecta los errores en el caso de que las reglas gramaticales sean violadas. Por ejemplo, un procedimiento muy común es la inclusión de reglas agramaticales (del término técnico en inglés buggy rules), que hacen posible que el parser procese una oración que contiene uno o más errores e identificarlos (Dodigovich, 2005; Heift \& Schulze, 2007). Sistemas más recientes de diagnóstico utilizan métodos estadísticos con grandes córpora con errores de alumnos de L2 para poder reconocer errores (Tschichold, 2003; Dodigovich, 2005). La ventaja de los enfoques con gramática de errores es que la respuesta al alumno puede ser muy específica. Pero la gran desventaja es que los errores individuales de los alumnos deben ser anticipados, es decir, cada error debe ser cubierto por una regla adecuada a ese error (Heift \& Schulze, 2007). Otra forma de realizar dicho análisis es a través de la técnica llamada cbunking, que sirve para la detección de ciertas identidades o secuencias específicas de texto, de esta forma esta técnica se usa para buscar errores morfológicos dentro de una oración mediante reglas similares a las buggy rules, pero que en este caso se centra en la búsqueda de secuencias de etiquetas específicas dentro de una oración que representa un error morfológico, como por ejemplo: una oración con error de tiempo o número. Los errores morfológicos son informados mediante una codificación del mismo. El análisis sintáctico utiliza técnicas de parsing basadas en gramáticas de contexto libre o de estructura de frase. En particular, se utiliza chart-parsing, que se diferencia de los otros métodos de parsing por la utilización de la programación dinámica, lo que la hace más eficiente en lenguas con ambigüedad, además evita el back-tracking o retroceso y previene de una explosión combinatoria. 
Esta gramática incluye reglas de errores sintácticos que corresponden principalmente a la estructura de la oración (por ejemplo, errores de orden, de omisión y adición de palabras entre otros, mediante buggy rules) y de esta forma los errores son detectados mediante la inspección del árbol generado por el parsing.

\subsection{Generación de Lenguaje Natural (GLN) y STI}

El área del PLN denominado Generación de Lenguaje Natural se entiende como la construcción o producción de textos a través de sistemas inteligentes con el objeto de responder a propósitos comunicativos (McDonald, 1992). El objetivo final es construir sistemas computacionales que produzcan textos coherentes en una o más lenguas (Lavid, 2005). En el ámbito de los STI, los generadores automáticos son un componente muy relevante en la medida que permiten interactuar con el estudiante en la lengua correspondiente facilitando de esta forma el proceso de andamiaje y apoyo en el aprendizaje de los contenidos y en la práctica de las habilidades lingüísticas, mejorando, de este modo, la competencia lingüística y comunicativa en lo que atañe a la precisión gramatical.

Los sistemas de GLN existentes en el ámbito de PLN descomponen el proceso de generación de un texto o enunciado en distintas tareas repartidas a lo largo de diferentes componentes o módulos. La tendencia más generalizada en la literatura especializada (Hovy \& Wanner, 1996; Reiter \& Dale, 2000) considera que el proceso de generación puede descomponerse en tres componentes o tareas principales: la macroplanificación, la microplanificación y la realización superficial. La macro y micro planificación textuales especifican el contenido y la estructura del dominio temático que se va a expresar. La realización superficial se ocupa de la forma del texto, es decir, la realización lingüística, y el formato del texto.

En lo que atañe al proceso de generación, se han desarrollado diferentes formalismos para representar y producir la lengua en los STI. Así, por ejemplo, en el área de diálogo entre agentes conversacionales, el objetivo fundamental puede ser que el sistema genere la retroalimentación adecuada, ante la respuesta del estudiante, para que este se percate de su error. Se trata de responder la pregunta ¿qué debe decir el sistema para que el estudiante logre responder correctamente? Para ello es necesario centrarse en la Planificación del Diálogo (PD) y en el Reconocimiento de Diálogo (RD). Este tipo de técnicas, basadas en métodos estadísticos tales como Condicional Random Fields (CRF) (Sutton \& McCallum, 2006), requieren de entrenamiento para poder ser aplicadas. El entrenamiento define en gran medida el cómo se hará la selección de la estrategia en un momento u otro. La interacción dialógica entre el estudiante y el STI también define el cómo debe realizarse la selección de estrategia de retroalimentación. Esto permite que la retroalimentación se ajuste al estudiante y al tipo de respuesta que este entregue. En el caso del modelo de diálogo, se requiere de un preprocesamiento previo tanto léxico como sintáctico lo que se logra a través de 
un etiquetado léxico (Part-of-speech tagging) y de analizadores sintácticos superficiales (chunkers). Estos últimos caracterizan grupos de palabras o pedazos de oraciones. Cada oración contiene en forma explícita o implícita una estructura que le da sentido. La estructura es consecuencia de las relaciones entre palabras individuales o entre partes de oraciones. Para descubrir dicha estructura se han utilizado tradicionalmente métodos estadísticos como cadenas de Markov (HMM) y modelos de lenguaje basados en N-gramas. Una vez que se ha encontrado el sentido de las oraciones, se busca la relación que puede haber entre ellas. Dicha relación define el contexto del diálogo, el objetivo tras este y permite la toma de turnos conversacionales estudiante-profesor.

Otro formalismo utilizado para GLN son las gramáticas de unificación de rasgos: bajo esta denominación se agrupan un conjunto importante de formalismos y teorías gramaticales que se caracterizan por utilizar rasgos en la descripción gramatical, por combinar y comprobar la información gramatical mediante una operación conocida como unificación. Existen distintas formulaciones de este tipo de gramáticas, la primera desarrollada es la Gramática de Unificación Funcional (Functional Unification Grammar o FUG) (Kay, 1979). Luego se desarrollaron: la Gramática Léxico-Funcional (Lexical-Functional Grammar o LFG) (Bresnan, 1982), la Gramática Categorial de Unificación (Categorial Unification Grammar o CUG) (Haddock, Klein \& Morrill, 1987); seguidas por la Gramática de Adjunción de Árboles (Tree Adjoining Gramar o TAG) (Joshi \& Schabes, 1992) y la Gramática Sintágmática Nuclear (Head Phrase Syntagmatic Grammar o HPSG) (Pollard \& Sag, 1987, 1994). A pesar de las diferencias entre ellas, las gramáticas de unificación y rasgos se basan en dos mecanismos principales:

1. La utilización de descripciones funcionales (FDs) o estructuras de rasgos para la representación de la información lingüística. Estas se constituyen en el mecanismo básico de representación de la información lingüística. Un FD simple es un conjunto de descriptores y un descriptor puede ser un conjunto de constituyentes, un patrón o un par compuesto por un atributo y un valor asociado y;

2. La utilización de una operación de unificación para el caso en que una entidad lingüística tenga dos descripciones funcionales diferentes.

El Sistema E-TUTOR desarrollado por Heift (2003) se basa en un modelo de la Gramática Sintágmática Nuclear (HPSG) para analizar, generar oraciones y producir conjuntos descriptores de frase. En este sistema, cada descriptor precisa una restricción gramatical particular (como por ejemplo, la concordancia sujeto-verbo), la presencia o ausencia en la oración de entrada y la actuación del estudiante en esta restricción. De esta forma, se organiza y conduce la producción de estrategias de feedback para apoyar los errores cometidos por los estudiantes. En la generación de estructuras de retroalimentación correctiva nuestro modelo de generador sigue el formalismo precisado por la gramática HPSG para orientar la realización superficial de las estrategias de feedback. 


\section{Arquitectura del STI ELE-TUTOR}

Acorde con la arquitectura clásica delimitada por Carbonell (1970), el STI ELETUTOR se estructura a partir de tres componentes básicos, como se muestra en la Figura 2:

1. El módulo del dominio, que considera los principios y parámetros relativos al enfoque metodológico de enseñanza Focalizado en la Forma (del inglés Focus on Form) en que se sustenta el desarrollo de contenidos, actividades, ejercicios y estrategias de feedback correctivo que el sistema proveerá al estudiante durante el proceso de enseñanza-aprendizaje de la lengua.

2. El módulo del estudiante, que contiene información acerca del estudiante (su conocimiento del dominio, su nivel de proficiencia en el Español como Lengua Extranjera, sus errores gramaticales más frecuentes, su estilo de aprendizaje, etc.).

3. El módulo del tutor que considera las capacidades y estrategias de enseñanza que el sistema tutorial evidenciará (como inteligentes) en su interación con el alumno, esto es, la capacidad de reconocer e identificar los errores de lengua que el estudiante cometa (analizador automático) como de aplicar estrategias de feedback correctivo efectivas (generador automático de feedback) y adecuadas al tipo de error, nivel de proficiencia y estilo de aprendizaje que el estudiante ha reflejado durante la ejecución de las actividades comunicativas.

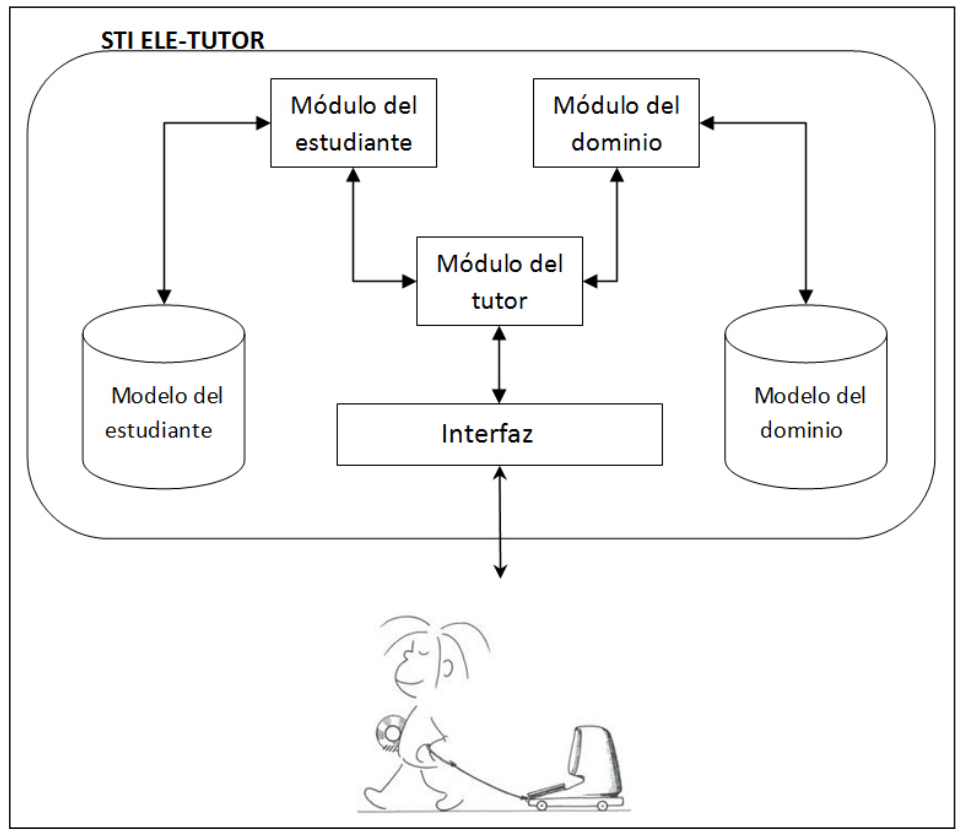

Figura 2. Arquitectura básica del STI ELE-TUTOR. 
Como se puede observar en la Figura 2 se representan modelos y módulos de ELETUTOR con funciones distintas, por un lado el modelo del estudiante y el modelo del dominio, que son las bases de datos encargadas de almacenar el conocimiento que mantiene el sistema del estudiante y del dominio respectivamente, y por otro lado, los módulos del estudiante, del dominio y del tutor, que representan los métodos implementados en el sistema, encargados de realizar las operaciones necesarias para mantener los modelos y para interactuar con el estudiante.

En la Figura 3, se observa la propuesta de integración de los tres módulos, ya conteniendo los elementos fundamentales (métodos), considerando los planos de conocimiento que hemos establecido anteriormente, y seguido de una tabla formal que establece las entradas y salidas de cada módulo (Tabla 1).

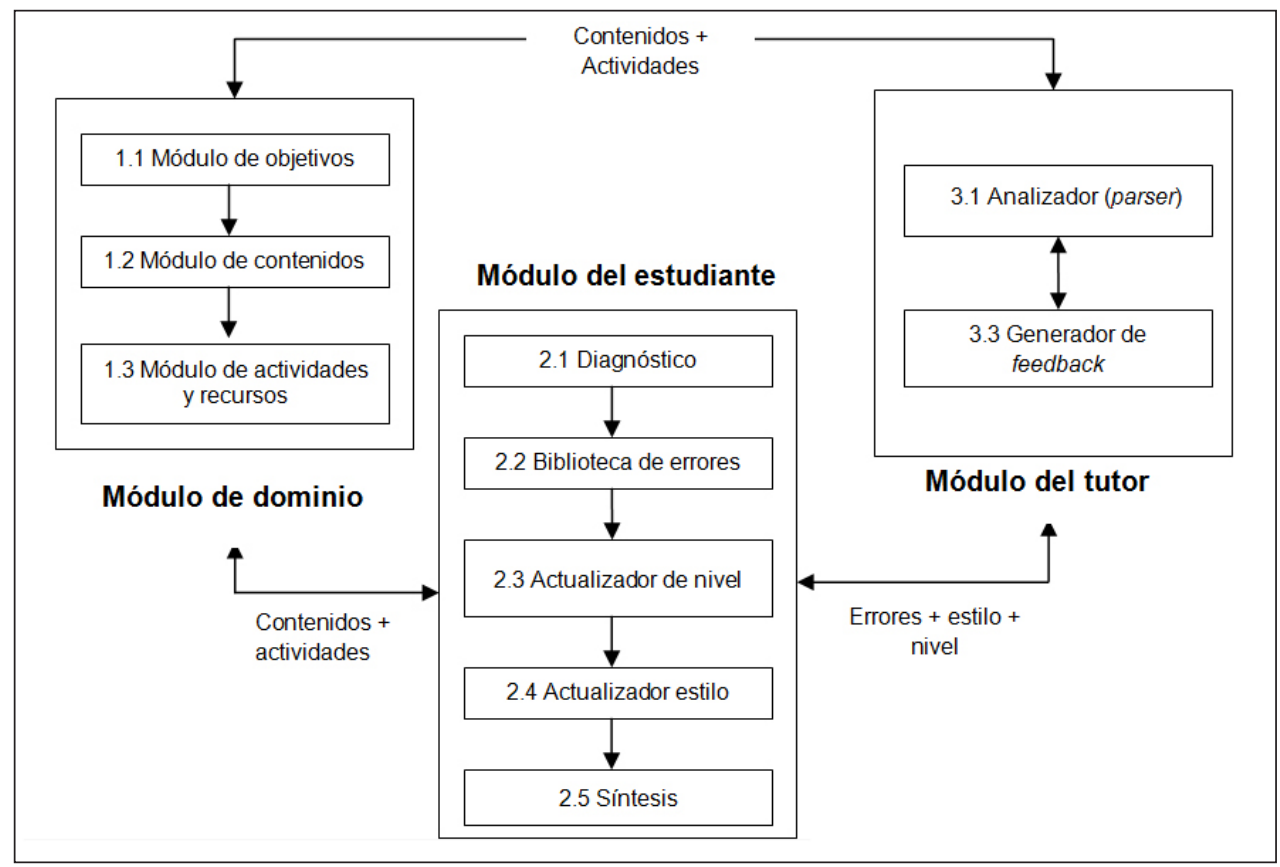

Figura 3. Métodos de cada módulo en la propuesta y mensajes entre ellos. 
Tabla 1. Entradas y salidas de los distintos módulos del sistema.

\begin{tabular}{|l|l|l|l|}
\hline Entrada Salida & $\begin{array}{l}\text { Módulo de } \\
\text { dominio }\end{array}$ & Módulo del estudiante & Módulo del tutor \\
\hline Módulo de dominio & ----------- & $\begin{array}{l}\text { contenidos y actividades } \\
\text { (ej. objetivos, materias, } \\
\text { test, consultas, etc.) }\end{array}$ & $\begin{array}{l}\text { contenidos y actividades } \\
\text { (ej. objetivos, materias, test, } \\
\text { consultas, etc.) }\end{array}$ \\
\hline Módulo del estudiante & ------------ & ---------- & $\begin{array}{l}\text { Conocimiento del alumno, } \\
\text { errores anteriores }\end{array}$ \\
\hline Módulo del tutor & ------------ & $\begin{array}{l}\text { errores cometidos por el } \\
\text { alumno }\end{array}$ & ------------ \\
\hline
\end{tabular}

Como se explicará detalladamente en las siguientes secciones, la Tabla 1 muestra cómo el módulo del dominio proporciona al módulo del estudiante y del tutor, los contenidos y actividades necesarias para llevar a cabo los procesos de actualización del modelo del alumno y de las actividades (preguntas y feedback) de los respectivos módulos. Lo mismo sucede con el módulo del estudiante, el cual proporciona al módulo del tutor el conocimiento del alumno y sus errores. A su vez, el módulo del tutor proporciona al módulo del estudiante los errores cometidos por este, para poder de esta forma actualizar el modelo del alumno.

\subsection{Módulo del dominio}

Como se señaló anteriormente, el enfoque de enseñanza de lengua que subyace al diseño de contenidos, actividades comunicativas y ejercicios gramaticales del sistema ELE-TUTOR corresponde al denominado técnicamente en el ámbito de la lingüística aplicada Enfoque en la Forma (del término técnico en inglés Focus on Form). En consecuencia, los criterios de selección y jerarquización tanto de las formas lingüísticas que son tratadas por el sistema (futuro simple y compuesto, condicional simple y compuesto, estilo indirecto) como de las estructuras de andamiaje retroalimentativo (claves metalingüísticas, elicitaciones y clarificaciones) que orientan el diálogo y la interacción colaborativa entre el tutor inteligente y los estudiantes se sustentan en los principios teórico-metodológicos del Enfoque en la Forma.

Ellis (1997) plantea que un Enfoque en la Forma se puede lograr de dos maneras. En primer lugar, las actividades se pueden diseñar para hacer que los estudiantes se comuniquen yal mismo tiempo centren su atención en propiedades formales específicas. En segundo lugar, los profesores pueden optar por proporcionar retroalimentación de los errores de los estudiantes durante el transcurso de actividades comunicativas. Esto ofrecería una manera de evitar la desventaja de enfocar separadamente en cada una de ellas. Es decir, un énfasis en la corrección de formas lingüísticas erróneas generalmente se asocia con un enfoque en la forma y la precisión. Sin embargo, en el caso de un enfoque extremo en el significado y la fluidez, encontramos una ausencia absoluta de corrección de las formas lingüísticas erróneas. Así, se puede considerar 
que el hecho de corregir desempeña un rol vital en el establecimiento de un enfoque ya sea en la forma y la precisión o en el significado y la fluidez.

ELE-TUTOR tiene por objetivo apoyar al estudiante en el nivel de proficiencia B2 (acorde con el Marco Común Europeo de Referencia para las lenguas o MCER (Consejo de Europa, 2002)) que se entiende como un nivel intermedio-avanzado, pues considera entre sus competencias comunicativas la comprensión y producción de textos complejos, tanto orales como escritos sin mayor esfuerzo. ELE-TUTOR cuenta por el momento y de manera prototípica con los contenidos de futuro simple y compuesto, condicional simple y compuesto y estilo indirecto correspondientes a contenidos gramaticales propios de este nivel de aprendizaje y que funcionan dentro del contexto de una plataforma tutorial (ver un ejemplo de ejercicio en la Figura 4). Con la finalidad de apoyar el aprendizaje de las formas lingüísticas mencionadas dentro de un contexto comunicativo (Pastor Cesteros, 2006; Doughty \& Varela, 2009), se ha elaborado una serie de actividades comunicativas y ejercicios gramaticales. Los ejercicios son específicamente de completación de oraciones (ver Figura 4) y respuestas a preguntas, todos ellos con más de tres palabras a completar y, dada la variedad de formas posibles en la entrada del usuario, tanto de formas correctas como incorrectas, hace necesario un reconocimiento a través de técnicas de PLN.

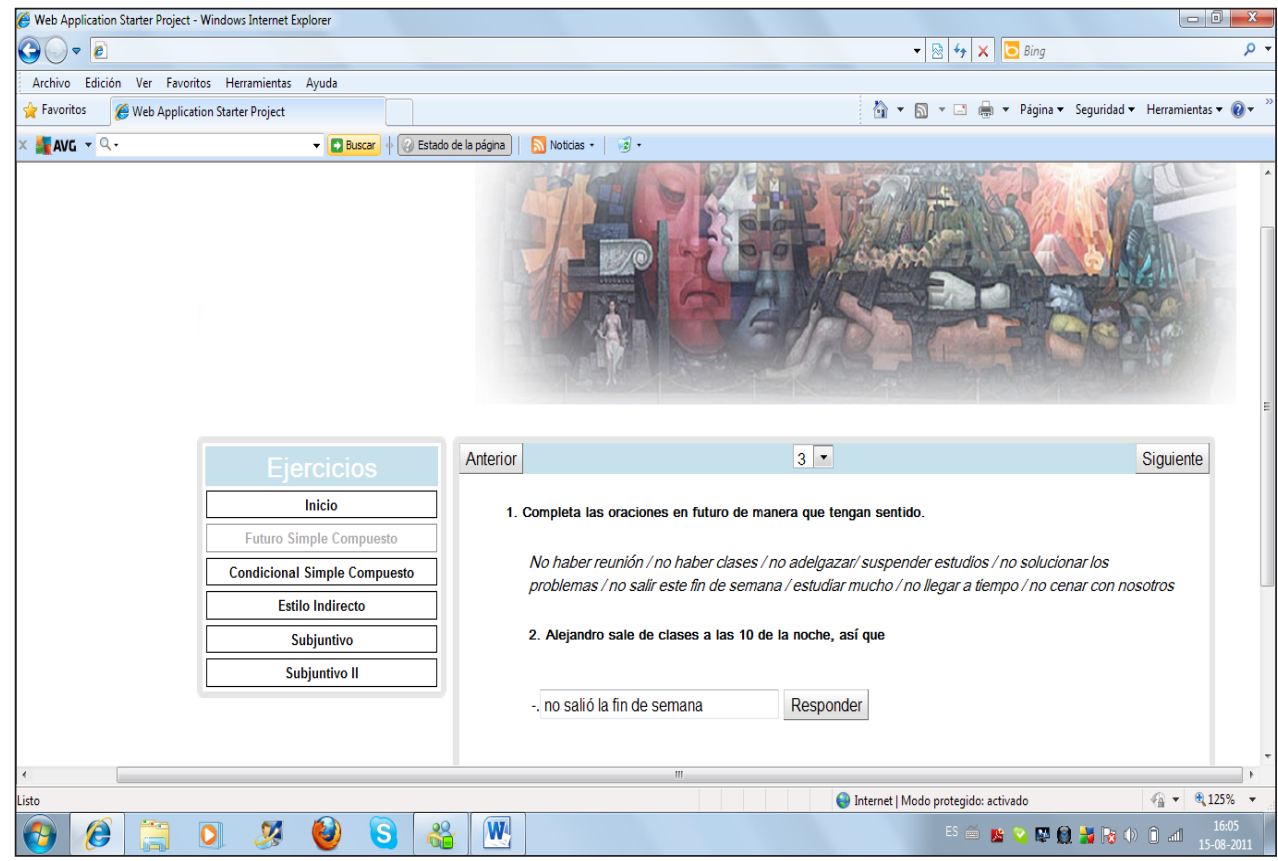

Figura 4. Ejemplo de ejercicio de completación focalizado en la forma gramatical de 'futuro'. 


\subsection{Módulo del estudiante}

Para lograr una articulación completa de los tres módulos que conforman el sistema ELE-TUTOR es relevante determinar: a) las características del alumno que considerará el modelo del estudiante; b) generar modelos del estudiante a partir de esas variables; c) determinar estrategias de enseñanza para cada tipo de estudiante modelado; y d) establecer un algoritmo que permita asignar una estrategia adecuada a cada estudiante.

Si se considera entonces que un STI tiene entre sus objetivos fundamentales la adaptabilidad, la cual está ampliamente determinada por la cobertura y precisión de la información contenida en el modelo de estudiante (Wenger, 1987), entonces una gran parte de las funciones que debe ejecutar el sistema para lograr este objetivo se encuentra en el modelo de estudiante. De este modo, modelar atributos adicionales del estudiante provee al sistema con más información y, en definitiva, al usuario con una experiencia más individualizada (Heift \& Schulze, 2007).

De acuerdo a lo anterior, el aporte específico del modelo de estudiante es, por una parte, obtener datos sobre las características del estudiante y almacenarlos en una base de datos para su posterior utilización: y por otra parte, actualizar constantemente esta información en caso de que cambie durante la interacción con el STI. Todos los datos almacenados en el modelo de estudiante son utilizados luego por el sistema para seleccionar una estrategia de feedback que mejor se adapte a las características del estudiante. El modelo del estudiante de ELE-TUTOR (como se observa en la Figura 5) recoge una serie de información con respecto a cada uno de los estudiantes que interactúan con el STI. En el modelo planteado, el sistema recibe información con respecto al estilo de aprendizaje, el nivel de proficiencia y el tipo de error que comete el estudiante, lo cual permite que el módulo tutor genere un feedback específico y adaptado a las características de cada estudiante. 


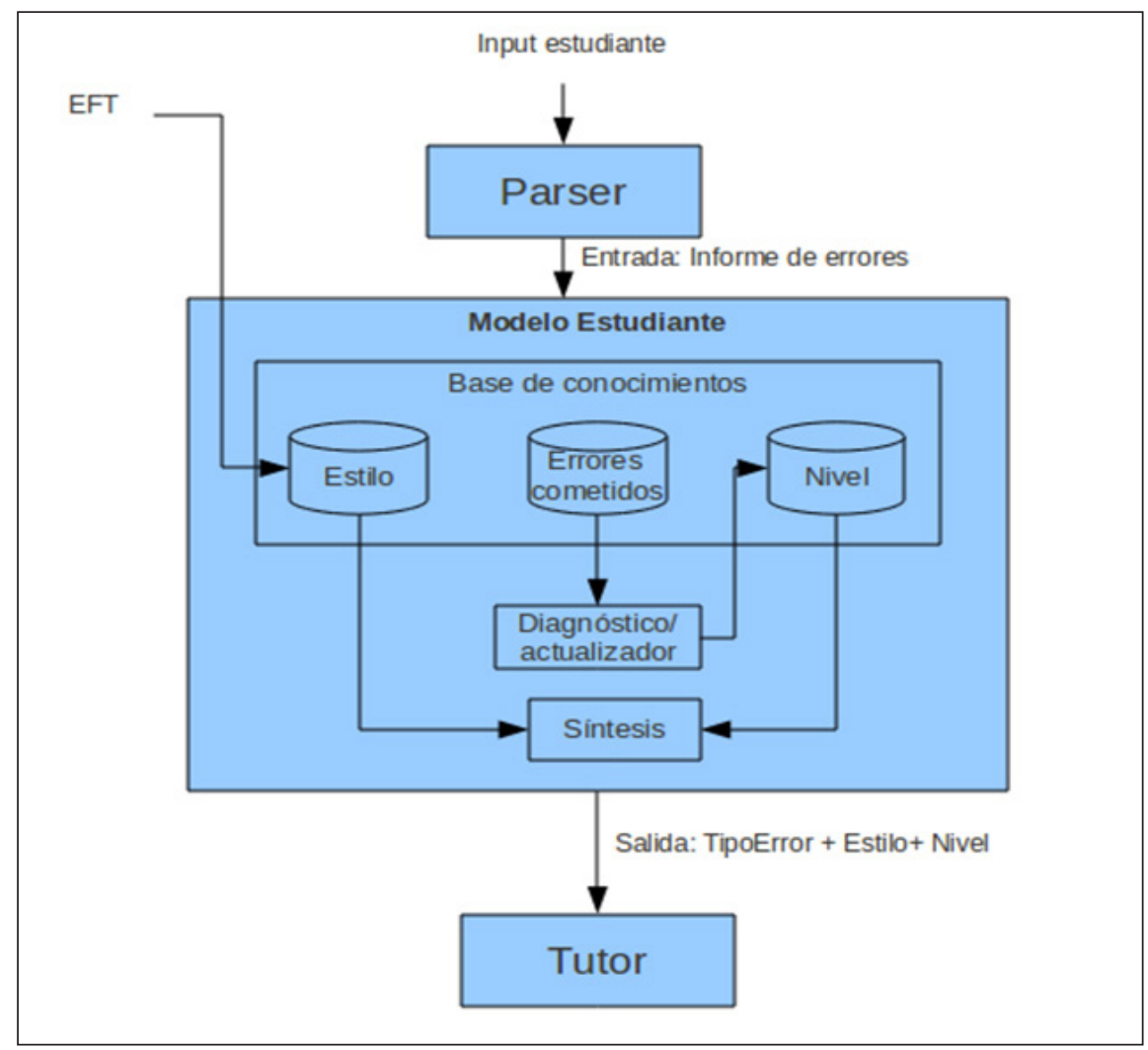

Figura 5. Modelo de estudiante: Componentes e integración con los otros módulos del STI.

Con respecto al estilo de aprendizaje del estudiante, se ha tomado en consideración para su determinación los conceptos de dependencia e independencia de campo del modelo de Witkin (1962) y Skehan (1998), el cual ha sido reelaborado constantemente en el área de la lingüística (sobre la evolución del concepto ver revisiones de Ellis, 1997; Skehan, 1998). Desde una perspectiva general, ambos conceptos se refieren a si un individuo tiende a separar los detalles del marco general o a observar las cosas más holísticamente (Lightbown \& Spada, 1999). Sin embargo, para el uso específico del concepto en el ámbito de la enseñanza de lenguas extranjeras, las definiciones de dependencia e independencia de campo aluden puntualmente a la tendencia del estudiante a aprender una lengua desde una perspectiva comunicativa o una centrada en la gramática, respectivamente (Willing, 1988).

Los conceptos de dependencia e independencia de campo determinarán el tipo de feedback correctivo que se enviará al estudiante cada vez que el sistema detecte un error, seleccionando estrategias más cercanas a un intercambio real de comunicación para la 'dependencia de campo' o bien otras más centradas en el análisis gramatical 
para los 'independientes de campo'. De este modo, la información referente al estilo de aprendizaje de los estudiantes se obtiene mediante un test de entrada, el Embedded Figures Test (EFT), diseñado específicamente para determinar la tendencia del estudiante en cuanto a la polaridad dependencia/independencia de campo (Witkin, 1962) y el cual es aplicado al estudiante por el mismo STI antes de que el estudiante comience con los ejercicios propuestos.

La consideración del nivel de proficiencia del estudiante en la selección del feedback correctivo obedece a diversos estudios (Ferreira et al., 2007; Heift, 2007). En ellos, se encuentra evidencia empírica que muestra cómo para cada nivel de proficiencia se necesita una estrategia diferente. Mientras Heift y Schulze (2007) hacen hincapié en el concepto de granularidad en el caso específico de la clave metalingüística, Ferreira et al. (2007) definen determinados tipos de feedback como idóneos para cada uno de los niveles. En el caso de este modelo, se propone una fusión de ambos enfoques, resultando en distintas estrategias de feedback (elicitación y clave metalingüística) que a la vez son granularizadas.

La información sobre el nivel de proficiencia del estudiante es especificada en ELE-TUTOR a través de la misma interacción con el STI, ya que cada error que se comete queda almacenado en la base de conocimientos del modelo de estudiante. Una vez que el estudiante completa una serie de ejercicios, el STI toma los datos almacenados hasta el momento y ejecuta un cálculo bayesiano que determinará el nivel de proficiencia del estudiante. Esto es, sobre el tipo de error cometido, el modelo del estudiante toma como entrada el informe de errores que el analizador arroja en forma de código cada vez que el estudiante ingresa un enunciado al sistema. Una vez que los errores son almacenados en la base de conocimiento, el sistema asigna un determinado peso a cada uno de los errores cometidos, para luego enviar la información al módulo diagnóstico/actualizador que ejecuta un cálculo bayesiano que permitirá conocer el nivel de proficiencia del estudiante. Esto se fundamenta en que una red bayesiana tiene la capacidad de calcular la probabilidad de un evento tomando variadas evidencias para este cálculo, ya que los tipos de ejercicios que se proponen al estudiante en el STI permiten una flexibilidad en las respuestas y, por lo tanto, la capacidad de cometer más de un error en cada pregunta. De este modo, se hace necesaria una forma de medición que considere varias evidencias en el cálculo; más aún, cada una de estas evidencias tiene un peso diferente, ya que los errores pueden ser más o menos representativos del nivel que se calcula.

El peso de cada uno de los errores se calcula tomando en cuenta la frecuencia de cada error en el estudio piloto con el fin de establecer qué errores corresponden al nivel B2. Los errores que presentaban una mayor frecuencia se estimaron como los que debían tener más peso, dado que un mayor número de errores implica una mayor dificultad. Así, cada uno de los ellos adquirió un valor diferente, con lo que la medición del nivel de proficiencia del estudiante se hace más preciso. 
De este modo, el sistema arroja una salida de tipo $\mathrm{E}=$ TipoError + EstAprend + NivEst. En cada caso, los valores por cada una de estas variables serán diferentes, lo que da la posibilidad al modelo tutor entregar al estudiante una estrategia de feedback adecuada a sus características. Por ejemplo, la Tabla 2 muestra las posibilidades en cuanto a los tipos de feedback que puede entregar el STI una vez que el modelo tutor recibe la salida del modelo de estudiante.

Tabla 2. Tipo de feedback entregado por ELE-TUTOR según nivel de proficiencia y estilo de aprendizaje del estudiante.

\begin{tabular}{|c|c|c|c|c|}
\hline & & \multicolumn{3}{|l|}{ Nivel de proficiencia } \\
\hline & & Inicial & Intermedio & Avanzado \\
\hline \multirow{2}{*}{$\begin{array}{l}\text { Dependiente } \\
\text { de campo }\end{array}$} & $\begin{array}{l}\text { Primer } \\
\text { intento }\end{array}$ & $\begin{array}{l}\text { Elicitación a nivel de } \\
\text { sintagma }\end{array}$ & $\begin{array}{l}\text { Elicitación a nivel de } \\
\text { sujeto-predicado }\end{array}$ & Clarificación \\
\hline & \begin{tabular}{|l} 
Segundo \\
intento
\end{tabular} & $\begin{array}{l}\text { Elicitación a nivel de } \\
\text { palabra }\end{array}$ & $\begin{array}{l}\text { Elicitación a nivel de } \\
\text { sintagma }\end{array}$ & $\begin{array}{l}\text { Elicitación a nivel } \\
\text { de sujeto-predicado }\end{array}$ \\
\hline \multirow{2}{*}{$\begin{array}{l}\text { Independien- } \\
\text { te de campo }\end{array}$} & $\begin{array}{l}\text { Primer } \\
\text { intento }\end{array}$ & $\begin{array}{l}\text { Clave metalingüística } \\
\text { a nivel de sintagma }\end{array}$ & $\begin{array}{l}\text { Clave metalingüística } \\
\text { a nivel de } \\
\text { sujeto-predicado }\end{array}$ & $\begin{array}{l}\text { Elicitación a nivel } \\
\text { de sujeto-predicado }\end{array}$ \\
\hline & $\begin{array}{l}\text { Segundo } \\
\text { intento }\end{array}$ & $\begin{array}{l}\text { Clave metalingüística } \\
\text { a nivel de palabra }\end{array}$ & $\begin{array}{l}\text { Clave metalingüística } \\
\text { a nivel de sintagma }\end{array}$ & $\begin{array}{l}\text { Clave } \\
\text { metalingüística a } \\
\text { nivel } \\
\text { sujeto-predicado }\end{array}$ \\
\hline
\end{tabular}

Finalmente, toda la información almacenada por el modelo de estudiante entrega al tutor una serie de posibilidades de feedback correctivo según un enunciado determinado escrito por un estudiante en particular. La Tabla 3 muestra una propuesta de enunciados concretos de feedback para mostrar al estudiante, en caso de que se cometa un error de tiempo en el verbo. 
Tabla 3. Enunciados específicos de feedback enviados por el STI una vez que el modelo tutor recibe la información del modelo de estudiante.

\begin{tabular}{|c|c|c|c|c|c|c|}
\hline & \multicolumn{3}{|c|}{ Nivel } & \multicolumn{2}{|c|}{ Estilo } & \multirow{2}{*}{ Output } \\
\hline & Ini & Int & $\mathrm{Av}$ & FD & FI & \\
\hline \multirow{6}{*}{$\begin{array}{l}\text { VT\%[tiempo } \\
\text { específico] }\end{array}$} & $\mathrm{X}$ & & & $\mathrm{X}$ & & $\begin{array}{l}\text { Reformula lo que aparece destacado: Alejandro sale de } \\
\text { clases a las } 10 \text { de la noche, así que "no salió la fin de } \\
\text { semana". }\end{array}$ \\
\hline & $\mathrm{X}$ & & & & $\mathrm{X}$ & $\begin{array}{l}\text { Has cometido un error de tiempo en el verbo de la } \\
\text { oración. }\end{array}$ \\
\hline & & $\mathrm{X}$ & & $\mathrm{X}$ & & $\begin{array}{l}\text { Reformula lo que aparece destacado: Alejandro sale de } \\
\text { clases a las } 10 \text { de la noche, así que "no salió la fin de } \\
\text { semana". }\end{array}$ \\
\hline & & $\mathrm{X}$ & & & $\mathrm{X}$ & Has cometido un error en el predicado de la oración. \\
\hline & & & $\mathrm{X}$ & $\mathrm{X}$ & & ¿Puedes reformular tu oración? No comprendo. \\
\hline & & & $\mathrm{X}$ & & $\mathrm{X}$ & $\begin{array}{l}\text { Reformula lo que aparece destacado: Alejandro sale de } \\
\text { clases a las } 10 \text { de la noche, así que "no salió la fin de } \\
\text { semana". }\end{array}$ \\
\hline
\end{tabular}

\subsection{Módulo del tutor}

El diseño del módulo del tutor se sustenta en los resultados de una serie de estudios empíricos (Ferreira 2003, 2006, 2007; Ferreira et al., 2007; Ferreira \& Kotz, 2010) que tenían por objetivo delimitar un modelo de tratamiento de los errores lingüísticos para un componente de estrategias de feedback correctivo de un STI para el español como LE.

Como se puede observar en la Figura 6, el módulo del tutor presenta dos componentes principales: un analizador y un generador de retroalimentación correctiva. La secuencia comienza con una respuesta del estudiante que contiene al menos un error de lengua. En el caso de que aparezca más de un error, es necesario tomar una decisión acerca de qué error debería ser tratado primero. El tratamiento de errores y generación de estrategias feedback correctivo en el contexto del módulo del tutor tiene en consideración aspectos tales como:

- La incorporación de estrategias de feedback correctivo de manera natural y auténtica dentro del enfoque de enseñanza focalizado en la forma (Focus on Form).

- La definición del grado de explicitación de las estrategias de feedback. Es necesario elegir entre estrategias de feedback correctivo que llaman la atención del estudiante hacia el error de manera no obstructiva (elicitación y clarificación explícita) y aquellas que dirigen la atención del estudiante al área del problema de manera más explícita (claves metalingüísticas).

- Tomar en cuenta el grado de efectividad de las estrategias de feedback correctivo de acuerdo con el tipo de error, el nivel de aprendizaje y el estilo de aprendizaje. 
- Determinar los tipos de errores que serán identificados por el analizador automático en el contexto de un proceso de tratamiento dirigido a formas de la lengua que se constituyen en excelentes candidatas para ser tratadas acorde con el modelo del estudiante.

En las secciones siguientes se describe con mayor detalle los dos componentes del módulo del tutor: el analizador automático (sección 3.3.1) y el generador automático (sección 3.3.2)

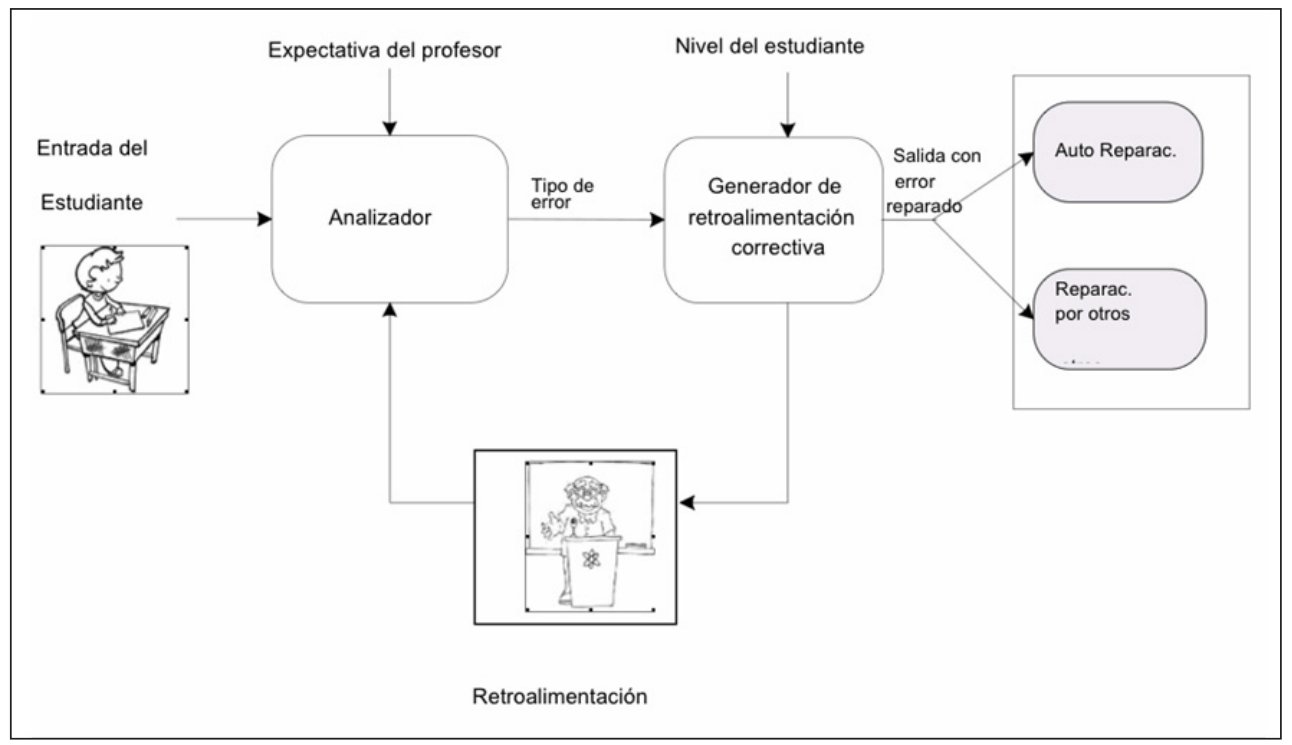

Figura 6. Módulo del tutor: Proceso del tratamiento del error y la generación de feedback correctivo del STI ELE-TUTOR.

\subsubsection{Descripción del analizador automático ELE-TUTOR}

La particularidad de este analizador es que debe procesar entradas erróneas y para ello, es necesario poder predecir los errores que puede cometer el usuario en un momento determinado de su aprendizaje y en un tópico gramatical específico. Para ello, se elaboró una taxonomía de errores de base teórica (Corder, 1981; Ellis, 1997; James, 1998) que se contrastó con un estudio observacional en clases tradicionales con el fin de obtener una información más acotada de los errores que podrían llegar a cometer alumnos de ELE con lengua materna inglés en su mayoría. Este análisis dio como resultado una taxonomía de errores acotada a la realidad muestral, pero que debe ser nuevamente ajustada para ser aplicada al tutorial con características particulares y con un modelo de alumno específico.

Debido a que el tratamiento de los errores cumple un rol preponderante, las especificaciones deben ser muy precisas. Debe tolerar, detectar y diagnosticar errores, como asimismo ser capaz de entregar una respuesta flexible en el reporte 
de los mismos. Un tema importante es el tratamiento de los errores múltiples, es decir, cuando una oración generada por el alumno contiene más de un error. Por un lado, es deseable que el programa sea capaz de detectar y explicar todos los errores, pero eso no significa que tenga que desplegar cada uno de los errores detectados. La ausencia de un mecanismo de filtro puede llegar a agobiar al alumno. Por ello, este sistema contiene una jerarquía de errores mediante el cual se despliega y se entrega una retroalimentación para el error más importante según esta jerarquía (Ferreira \& Kotz, 2010).

\section{3·3.1.1. La gramática formal y la gramática de errores}

Para el funcionamiento del analizador, es necesario elaborar una gramática formal, es decir, un conjunto de reglas de formación de frases que el analizador reconocerá. Para el caso del parser del ELE-TUTOR se construyó una gramática del tipo libre de contexto. Estas gramáticas, que se denominan también gramáticas de tipo 2 de Chomsky o gramáticas independientes del contexto, son las que generan los lenguajes libres o independientes del contexto; es decir, que en el lado izquierdo de una producción pueden aparecer el símbolo inicial $(\mathrm{O})$ o un símbolo no terminal y en el lado derecho cualquier cadena de símbolos terminales y/o no terminales de longitud mayor o igual que 1 (Lavid, 2005; Heift \& Schulze, 2007; Jurafsky \& Martin, 2008).

Además, está previsto una gramática formada por un conjunto de reglas agramaticales (buggy rules), lo que permite que el parser procese una oración que contiene uno o más errores e identificarlos. Los errores previstos corresponden a errores sintácticos y morfológicos principalmente a la estructura de la oración (por ejemplo, errores de concordancia, de orden, de omisión y adición de palabras entre otros) y de esta forma los errores son detectados mediante la inspección del árbol generado por el parsing.

\subsubsection{Análisis morfológico y etiquetado de oraciones}

Para que una secuencia pueda ser analizada por el analizador a cada unidad léxica debe asignársele una etiqueta dentro de un conjunto de etiquetas seleccionado. El conjunto de etiquetas de este sistema se basa en las etiquetas creadas por el grupo EAGLES (2011) para la anotación morfosintáctica de lexicones y corpus de lenguas europeas. Dependiendo de la lengua hay atributos que no están especificados y, por lo tanto, hay que crear etiquetas ad hoc. También puede ocurrir el caso contrario, que se cuente con etiquetas que para el corpus no se consideran relevantes y no haya que incluirlas. A modo de ejemplo, para este analizador en particular, hubo que crear etiquetas para diferenciar las preposiciones, ya que dentro de la lista de etiquetas propuestas solo se ofrece una etiqueta para todas las preposiciones (SPS00) y, dado que el dominio de las preposiciones es un tópico importante en la enseñanza/aprendizaje del español, fue necesario crear una etiqueta para diferenciar cada preposición. 
Para el etiquetado del corpus de entrenamiento elaborado a mano, se tomó como base el etiquetador probabilístico Freeling 2.0 (que se encuentra disponible en la web en su versión demo). El etiquetado de la entrada de los alumnos se realiza con la técnica de N-gramas, más precisamente tri-gramas; es decir, por cada palabra de la oración ingresada el etiquetador revisa los dos contextos precedentes a la unidad a etiquetar y elige la etiqueta más probable. Luego busca en el corpus la misma palabra, revisa el contexto y 'memoriza' la palabra con su contexto y etiqueta asociada.

\subsubsection{Análisis sintáctico}

Para el análisis sintáctico se usa la técnica de chart-parsing, que se diferencia de otros métodos de análisis por la utilización de la programación dinámica, lo que mejora la eficiencia en lenguas ambiguas, además evita el back-tracking o retroceso y previene de una explosión combinatoria. Además, para optimizar el procesamiento se utiliza el método de chunking, que es una técnica que se usa para la detección de determinadas identidades o secuencias específicas de texto útiles para el análisis. Este analizador cuenta con un chunker de frases nominales, dado que estas frases requieren de un mayor procesamiento y, además, son más abundantes, ya que, por ejemplo, una frase preposicional contiene una frase nominal.

En la Figura 7 se precisan las distintas etapas del procesamiento sintáctico de la entrada "*no salió la fin de semana" realizada por el analizador automático ELETUTOR correspondiente al ejercicio de futuro en el cual el estudiante debe completar el enunciado "Alejandro sale de clases a las 10 de la noche, así que ...."*no salió la fin de semana" (ver Ejercicio en Figura 5).

Como se puede apreciar, el sistema reconoce dos errores; el primer error detectado no corresponde a un error en el sentido de la estructura de la frase, sino a un incumplimiento en los requerimientos del ejercicio que, en este caso, exige una oración en tiempo futuro; el segundo error, en cambio, es un error de concordancia entre el determinante 'la' y el sustantivo 'fin'. El modelo presenta una serie de pasos a seguir desde el momento que se introduce el enunciado hasta la salida del informe de errores. El alumno-usuario ingresa un enunciado como respuesta a un ejercicio que es etiquetado por un etiquetador de partes del habla (PoS Tagging, Part-of-speech tagging o simplemente POST) probabilístico con una técnica de 'tri-gramas'. La oración etiquetada es comparada con las del corpus de entrenamiento buscando coincidencias y analizada en tanto sus rasgos morfológicos y sintácticos. Para el análisis morfológico se utiliza la técnica de chunking de frases nominales para la detección de secuencias específicas en el texto y, de esta forma, encontrar errores. 


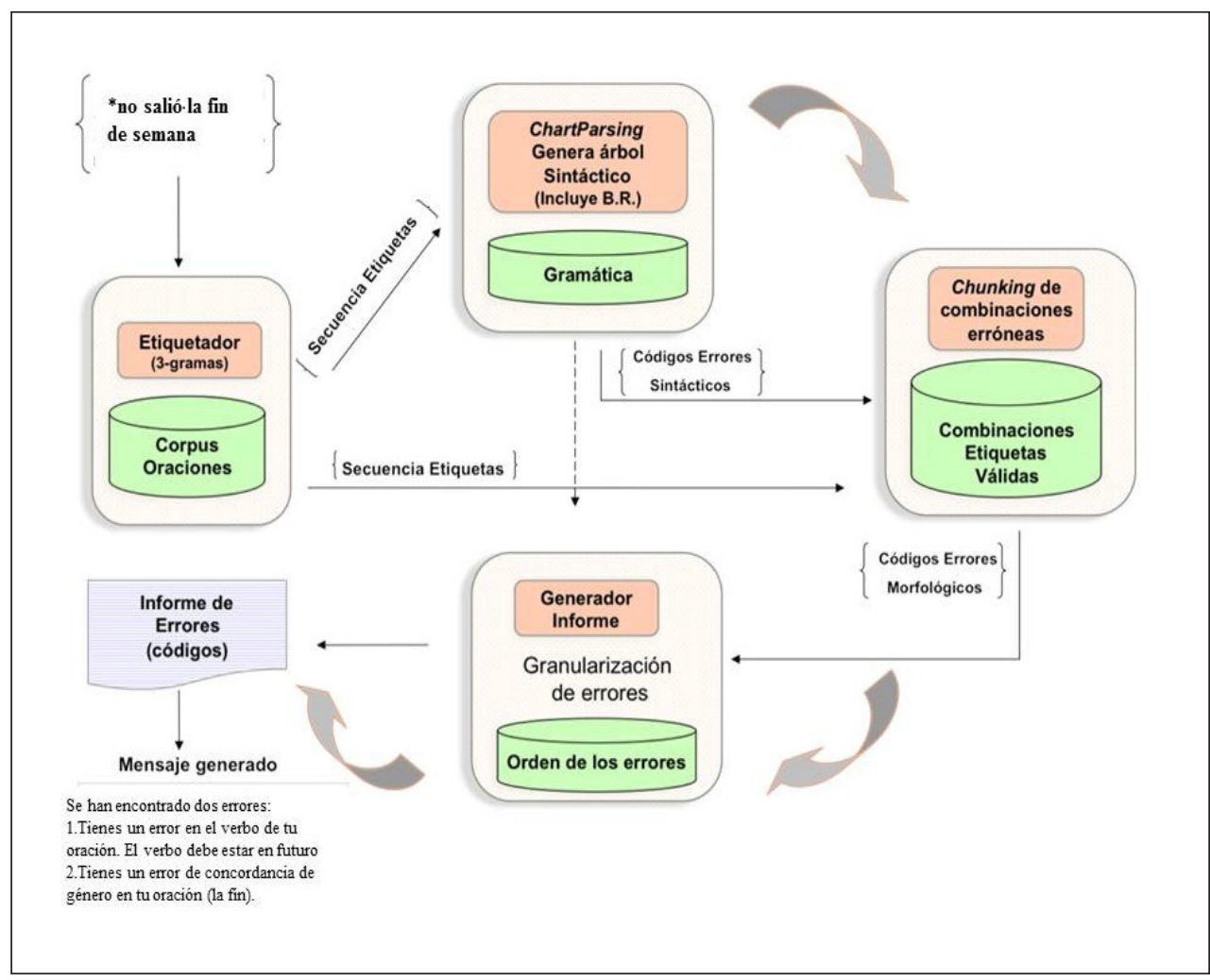

Figura 7. Modelo del parser ELE-TUTOR mostrando el procesamiento de la entrada de un alumno: "no salió la fin de semana".

De manera simultánea se realiza el análisis sintáctico mediante un chart-parsing, que es más eficiente para resolver problemas de ambigüedad muy frecuentes en lenguas como el español. Este parser opera con una gramática de contexto libre del tipo de estructura de frase que es ampliamente utilizada para el PLN (Lavid, 2005; Heift \& Schulze, 2007; Jurafsky \& Martin, 2008) y, como se trata de un parser que debe procesar entradas erróneas, incluye una gramática con reglas de errores (buggy rules o malrules) que corresponden a errores que los alumnos de L2 cometen con más frecuencia en un determinado nivel de aprendizaje la lengua; en este caso se trata del nivel B2 según el Marco Común Europeo de Referencia para las lenguas. Esta gramática contempla el tratamiento de errores de estructura de la oración (por ejemplo, errores de orden, omisión y adición) y errores en términos de categorías lingüísticas, basadas en el ítem lingüístico que es afectado por el error (errores en los verbos, en las preposiciones, etc.).

Una vez detectados los errores, se genera un informe que da cuenta de ellos en orden según una jerarquía definida previamente que, a su vez, sirve de input para el generador de feedback. 


\subsubsection{Generador de feedback correctivo ELE-TUTOR}

Una vez que el sistema ELE-TUTOR identifica un error a través de su analizador automático, debe seleccionar una estrategia de feedback correctivo ad-boc a dicho tipo de error y producirla en español por medio del generador automático. El feedback correctivo generado puede ser seguido por diferentes tipos de respuestas por parte de los estudiantes:

1. Una respuesta inmediata que contiene el error reparado ya sea por autoreparación o por reparación del sistema. Esto indica que el estudiante ha notado el error, por ende, la respuesta reparada se constituye en un indicio de mejoramiento en el aprendizaje. Una expresión reformulada por parte del estudiante da indicios de que la correlación entre la forma del estudiante y la forma final se ha notado, ello implica un paso hacia la adquisición (Lightbown, 1998).

2. Una respuesta que todavía contiene el error. Esto puede ocurrir porque el estudiante no ha notado la forma correcta provista por el sistema o bien porque el estudiante no tiene el conocimiento previo necesario para auto-corregir su error. En estos casos, el tutor trata el error sin reparación con una estrategia de feedback alternativa (elicitación o clarificación).

3. Una respuesta en la cual el estudiante repara el error original, pero su enunciado presenta un nuevo error. En este caso, el tutor selecciona una estrategia metalingǘstica acorde con el tipo de error gramatical presentado y el nivel de proficiencia del estudiante.

En la Figura 8 presentamos el diseño del Generador Automático de ELETUTOR en el contexto del módulo del TUTOR del sistema ELE-TUTOR. Como ya fue descrito en la sección anterior, las entradas que considera el módulo tutor corresponden a la información aportada por el módulo dominio (contenido y tipos de actividad) y por el módulo del estudiante, esto es, su nivel de proficiencia y su estilo de aprendizaje. Dicha información es útil tanto para el analizador como para el generador del módulo tutor. 


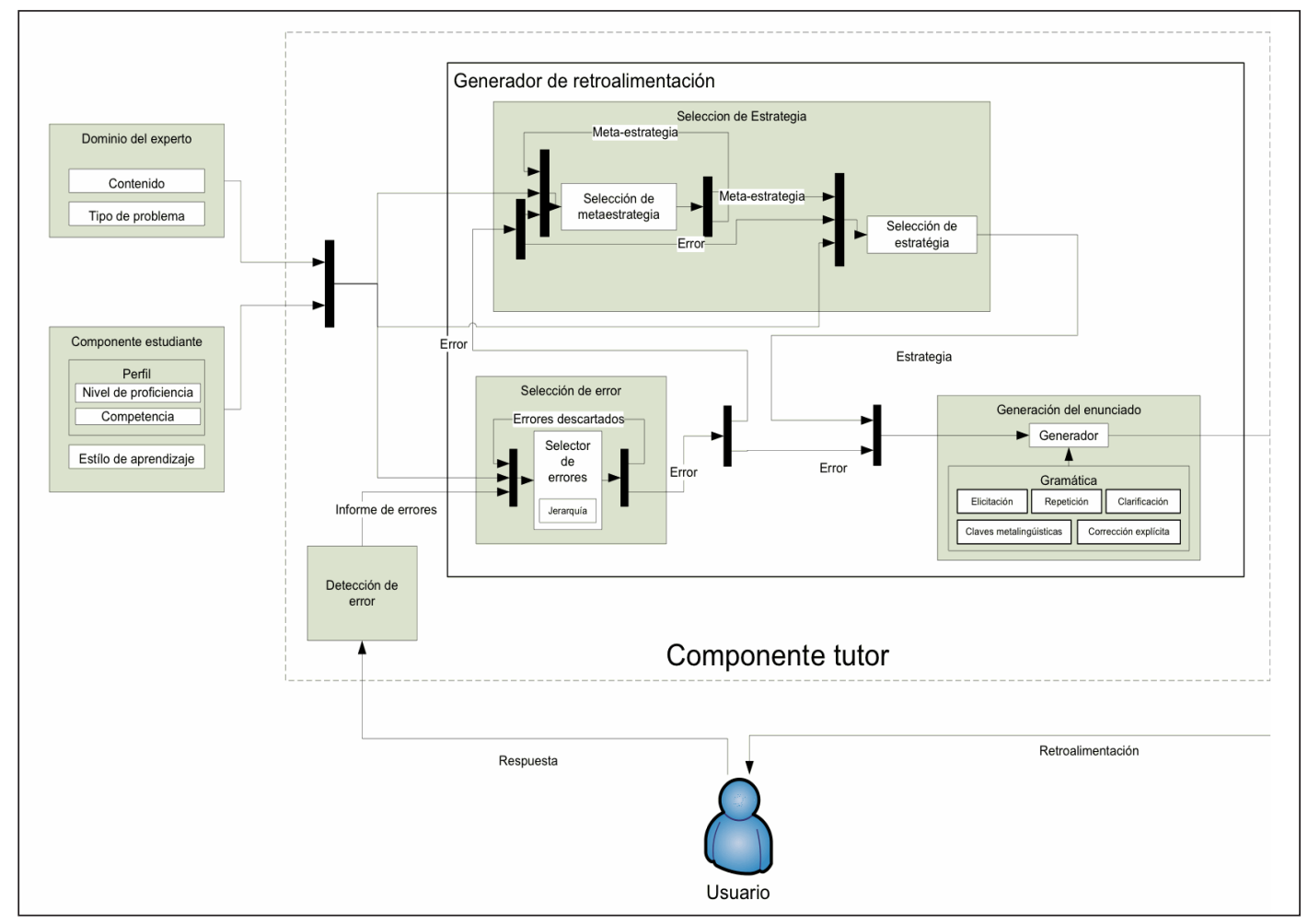

Figura 8. Modelo del generador del STI ELE-TUTOR.

Ahora bien, hasta aquí en la descripción del módulo del tutor hemos precisado que la tarea principal es identificar el tipo de error gramatical (basado en la focalización en la forma) que el estudiante presenta en su enunciado-respuesta a la actividad lingüística desplegada por el sistema. La identificación del error permite tratar de manera individualizada y focalizada esta problemática lingüística con el objeto de dar una estrategia correctiva más acorde con el tipo de error, permitiendo de esta manera apoyar el proceso de precisión lingüística de manera más efectiva. Es en esta parte del procesamiento del lenguaje donde se requiere del generador automático el cual debe en primer lugar llevar a cabo la tarea de seleccionar de un conjunto de estrategias correctivas (clave metalingüística, elicitación o clarificación) el tipo más apropiado para tratar el error que ha sido identificado y priorizado jerárquicamente por el analizador automático, todo ello en el contexto de la actividad y acorde con las caraterísticas registradas en el modelo del perfil del estudiante.

En consecuencia, la identificación y/o selección del tipo de error (cuando hay más de uno en la respuesta del estudiante) y la selección del tipo de estrategia constituyen las entradas para el subcomponente del generador automático que debe producir el enunciado en cuestión correspondiente al feedback correctivo que debe desplegar el sistema ELE-TUTOR para que el alumno note su error y lo repare, mejorando así su precisión gramatical. 
El generador del enunciado basado en el modelo de gramática de unificación de rasgos HSPG analiza, produce un conjunto de descriptores de frases y genera un enunciado correspondiente a la estructura superficial de la estrategia de feedback correctivo seleccionada. De esta manera, se estructura y coordina la generación automática de enunciados correspondientes a estrategias de feedback correctivo para apoyar el tratamiento de los errores que han sido reconocidos, seleccionados y tratados de manera focalizada e individualizada por el módulo TUTOR del sistema ELE-TUTOR.

\section{Comentarios finales y proyecciones de la investigación}

El artículo ha centrado su atención en el diseño de la arquitectura de un sistema tutorial inteligente para la Enseñanza del Español como LE: ELE-TUTOR. En general, la fundamentación teórica y empírica que subyace a la estructura y articulación de de cada uno de los módulos que componen el STI ponen de manifiesto la viabilidad de construir un sistema de tales características permitiendo con ello la identificación de errores gramaticales y el andamiaje de feedback correctivo necesario para apoyar de manera efectiva (individual y focalizada) el proceso de aprendizaje del español como L2 en el contexto metodológico de enseñanza focalizado en la forma.

Nuestro enfoque de investigación ha sido enriquecido por la investigación de diferentes disciplinas, incluyendo la Adquisición de Segundas Lenguas (ASL), los STI en contextos procedimentales, y los STI para el área de LE. Estas diversas perspectivas conducen a responder una pregunta general acerca de cómo los STI para LE pueden contribuir a aliviar las limitaciones o desventajas observadas en la modalidad presencial de clases tradicionales ya sea proveyendo mayor oportunidad de interacción a los estudiantes durante la clase o bien tratando los errores de los estudiantes con adecuadas y efectivas estrategias de feedback correctivo, como aquellas que apelan a que ellos sean capaces de resolver por sí mismos sus errores de lengua.

La metodología propuesta se sustenta en un modelo que concibe la enseñanza de la lengua asistida por el computador. En este contexto, se han delimitado los componentes necesarios básicos para desarrollar un STI que apoye la enseñanza de LE. En nuestro modelo ELE-TUTOR se ve superada una de las principales debilidades que presentan los sistemas CALL tradicionales que apoyan la enseñanza de la lengua, esto es, el uso de un feedback correctivo general y no contextualizado al tipo de error que el estudiante comete, ello repercute en una ayuda poco efectiva para mejorar la competencia lingüística y precisión gramatical. Con respecto al modelo del alumno, cabe destacar la consideración que se realiza en ELE-TUTOR de la información que se va adquiriendo de cada alumno que interactúa con el sistema, esta información es almacenada en bases de datos, considera tanto datos psicológicos (estilo de aprendizaje), como errores y es considerado como dato para que el tutor determine la mejor estrategia de enseñanza para cada alumno en forma personalizada. 
El sustento teórico y enfoque de enseñanza aprendizaje de lenguas en que se basa el modelo, esto es, enfoque en la forma, ha sido fundamentado en profundidad con el objeto de proveer las características diferenciadoras que hacen factible una mayor efectividad en la modalidad tutorial que se sugiere. El trabajo interactivo de los estudiantes en función de la resolución de una tarea, actividad o ejercicio focalizado en la forma mediatizado por un STI facilita la práctica de las habilidades lingüísticas que los estudiantes de lenguas deben mejorar y desarrollar. Los STI para LE pueden contribuir a aliviar las limitaciones o desventajas observadas en la modalidad presencial de clases tradicionales tratando los errores de los estudiantes con adecuadas y efectivas estrategias de feedback correctivo. Esto permite la individualización en la atención y selección de las problemáticas gramaticales, el respeto por los programas internos de los sujetos y las modificaciones en el tiempo y la forma de enseñanza. Con el objeto de continuar con la implementación y evaluación empírica de los distintos módulos de este sistema estamos diseñando una interfaz para llevar a cabo experimentos longitudinales con el propósito de probar la efectividad de ELE-TUTOR tanto a nivel de diseño (arquitectura) como de implementación y operacionalización en la Enseñanza del Español como LE (http://tigre. cfrd.cl/ parser/Tiae.php). 


\section{REFERENCIAS BIBLIOGRÁFICAS}

Amaral, L. \& Meurers, D. (2011). On Using Intelligent Computer-Assisted Language Learning in Real-Life Foreign Language Teaching and Learning. ReCALL, 23(1), 4-24.

Bresnan, J. (1982). The mental representation of grammatical relations. Cambridge: MIT Press.

Carbonell, J. R. (1970). AI in CAI: An Artificial Intelligence approach to Computer Assisted Instruction. IEEE transaction on Man Macbine System, 11(4), 190-202.

Consejo de Europa (2002). Marco Común Europeo de Referenciapara las Lenguas: Aprendizaje, Enseñanza, Evaluación [en línea]. Disponible en: http://cvc.cervantes.es/ ensenanza/biblioteca_ele/marco/

Corder, P. (1981). Error analysis and interlanguage. Oxford: University Press.

Chi, M., Soler, S., Jeong, H., Yamauchi, T. \& Hausmann, R. (2001). Learning from human tutoring. Cognitive Science, 25, 471-533.

Dodigovic, M. (2005). Artificial intelligence in second language learning: Raising error awareness. Clevedon: Multilingual Matters Ltd.

Doughty, C. \& Varela, E. (2009). Atención comunicativa a la forma. En C. Dougthy \& J. Williams (Eds.), Atención a la forma en la adquisición de segundas lenguas en el aula (pp. 127-152). Madrid: Edinumen.

EAGLES. Expert Advisory Group on Language Engineering Standards [en línea]. Disponible en: http://nlp.lsi.upc.edu/freeling/doc/userman/parole-es.pdf

Ellis, R. (1997). The study of second language acquisition. Oxford: Oxford University Press. Ferreira, A. (2003). Feedback strategies for second language teaching with implications for Intelligent Tutorial Systems. Tesis doctoral, Universidad de Edimburgo, Edimburgo, Reino Unido.

Ferreira, A. (2006). Estrategias efectivas de feedback positivo y correctivo en Español como Lengua Extranjera. Revista Signos. Estudios de Lingüistica, 39(62), 309406.

Ferreira, A. (2007). Estrategias efectivas de feedback correctivo para el aprendizaje de lenguas asistido por computadores. Revista Signos. Estudios de Lingüística, 40(65), 521-544.

Ferreira, A., Moore, J. \& Mellish, C. (2007). A study of feedback strategies in foreign language classrooms and tutorials with implications for Intelligent ComputerAssisted language learning systems. International Journal of Artificial Intelligence in Education, 17(4), 389-422.

Ferreira, A. \& Kotz, G. (2010). ELE-TUTOR Inteligente: Un analizador computacional para el tratamiento de errores gramaticales en Español como Lengua Extranjera. Revista Signos. Estudios de Lingüistica, 43(73), 211-236.

Free Ling 2.2. [en línea]. Disponible en: http://nlp.lsi.upc.edu/freeling/demo/demo. php

Graesser, A., Person, N. \& Magliano, J. (1995). Collaborative dialog patterns in naturalistic one-to-one tutoring. Applied Cognitive Psychology, 9, 495-522. 
Granger, S. (2003). Error-tagged Learner Corpora and CALL: A promising synergy. CALICO Journal, 20(3), 465-480.

Haddock, N. J., Klein, E. \& Morrill, G. (1987). Categorial Grammar, Unification Grammar, and Parsing. Technical Report No. EUCCS/WP-1. Centre for Cognitive Science, Edimburgo: Universidad de Edimburgo.

Heift, T. (2003). Multiple learner errors and feedback: A challenge for ICALL systems. CALICO Journal, 20(3), 549-560.

Heift, T. \& Schulze, M. (2007). Errors and intelligence in computer-assisted language learning. Parsers and Pedagogues. Nueva York: Routledge.

Hendrickson, J. (1979). Evaluating spontaneous communication trough systematic error analysis. Foreign Language Annals, 12(5), 357-364.

Holland, M., Kaplan, J. \& Sams, M. (1995). Intelligent language tutors: Theory shaping technology. Nueva Jersey: Lawrence Erlbaum.

Hovy, E. H. \& Wanner, L. (1996). Managing Sentence Planning Requirements. Ponencia presentada en el Workshop on New Directions in Planning and Natural Language Generation at the 12th European Conference on Artificial Intelligence, Hungría.

James, C. (1998). Error in Language Learning and Use. Exploring Error Analysis. Harlow: Pearson Education Limited.

Joshi, A. K. \& Schabes, Y. (1992). Tree-adjoining grammars and lexicalized grammars. En M. Nivat \& A. Podelski (Eds.), Tree Automata and Languages (pp. 409-431). Amsterdam: Elsevier Science.

Jurafsky, D. \& Martin, J. (2008). Speech and Language Processing. An Introduction to Natural Language Processing, Computational Linguistics, and Speech Recognition. Nueva Jersey: Prentice Hall.

Kay, M. (1979). Functional Grammar. Ponencia presentada en el 5th meeting of the Berkeley Linguistics Society, Berkeley Linguistics Society, California, USA.

Lavid, J. (2005). Lenguaje y nuevas tecnologias. Nuevas perspectivas, métodos y herramientas para el lingüista del siglo XXI. Madrid: Ediciones Cátedra.

Levin, L. \& Evans, D. (1995). ALICE-chan: A case study in ICALL theory and practice. En V. Holland, J. Kaplan \& M. Sams (Eds.), Intelligence Language Tutors: Theory Shaping Technology (pp. 77-98). Hillsdale, N.J.: Lawrence Erlbaum.

Lightbown, P. (1998). The importance of timing in focus on form. En C. Doughty \& J. Williams (Eds.), Focus on form in classroom second language acquisition (pp. 177196). Cambridge: Cambridge University Press.

Lightbown, P. \& Spada, N. (1999). How languages are learned. Oxford: Oxford University Press.

McDonald, D. (1992). Natural Language Generation. En C. Stuart Sapiro (Ed.), Encyclopedia of Artificial Intelligence (pp. 983-997). Nueva York: John Wiley and Sons.

Nagata, N. (2002). BANZAI: Computer Assisted Sentence Production Practice with Intelligent Feedback. Ponencia presentada en el Third International Conference on Computer- Assisted System for Teaching and Learning Japanese, San Diego, USA. 
Pastor Cesteros, S. (2006). Aprendizaje de Segundas Lenguas: Lingüistica Aplicada a la Enseñanza de Lenguas. Alicante: Publicaciones de la Universidad de Alicante.

Pollard, C. \& Sag, I. A. (1987). Information-based Syntax and Semantics. Volume I: Fundamentals. Stanford: CSLI.

Pollard, C. \& Sag, I. A. (1994). Head-driven phrase structure grammar. Chicago: Chicago Press.

Reiter, E. \& Dale, R. (2000). Building Natural Language Generation Systems. Cambridge: Cambridge University Press.

Skehan, P. (1998). A cognitive approach to language learning. Oxford: Oxford University Press.

Schulze, M. (2008). AI in CALL: Artificially Inflated or Almost Imminent? CALICO Journal, 25(3), 510-527.

Sutton, C. \& McCallum, A. (2006). An introduction to conditional random fields for relational learning. En L. Getoor \& B. Taskar (Eds.), Introduction to statistical relational learning (pp. 1-35). Cambridge: MIT Press.

Tschichold, C. (2003). Lexically driven error detection and correction. CALICO Journal, 20(3), 549-559.

Wenger, E. (1987). Artificial Intelligence and Tutoring Systems: Computational and Cognitive Approaches to the Communication of Knowledge. Los Altos, CA: Morgan Kaufmann.

Willing, K. (1988). Learning styles in adult migrant education. Adelaide, South Australia: National Curriculum Research Council.

Witkin, H. A. (1962). Psychological differentiation: Studies of development. Nueva York: Wiley.

\section{NOTA}

1 La arquitectura del Sistema Tutorial Inteligente para el Español como Lengua Extranjera que se presenta en este artículo se ha desarrollado en el contexto del proyecto de investigación FONDECYT 1110812 "Un Sistema Tutorial Inteligente para la focalización en la forma en la enseñanza del español como lengua extranjera". 